\title{
Studies of light mesons at BELLE
}

\author{
B.A.Shwartz $z^{1,2, a}$ \\ for the Belle collaboration \\ ${ }^{1}$ Budker Institute of Nuclear Physics, 630090 Novosibirsk, Russia \\ ${ }^{2}$ Novosibirsk State University, 630090 Novosibirsk, Russia
}

\begin{abstract}
Studies of light mesons in experiments with the Belle detector at the KEKB B-factory are described. Three types of hadron production processes are discussed: two-photon production, production via initial state radiation and hadronic tau lepton decays.
\end{abstract}

\section{Introduction}

Experiments with the Belle detector [1] at the asymmetricenergy $e^{+} e^{-}$KEKB collider [2] were performed from 1999 to 2010 in the centre-of-mass energy range within the $\Upsilon$-meson family. In these experiments the KEKB collider achieved the world highest collider luminosity, $2.1 \times 10^{34} \mathrm{~cm}^{-2} \mathrm{~s}^{-1}$. The total integrated luminosity collected by Belle is more than $1000 \mathrm{fb}^{-1}$. The primary goal of the Belle experiment was to discover $\mathrm{CP}$ violation (CPV) in $\mathrm{B}$ meson decays and to measure the parameters of CPV. This was achieved in 2001, when the time-dependent $\mathrm{CP}$ asymmetry was observed in the decay $B^{0} \rightarrow J / \Psi K^{0}$ decay [3]. However, besides of the main task, a lot of other important results were obtained. So a wide research area become possible because of the clean event environment and well-defined $e^{+} e^{-}$initial state as well as high luminosity and general-purpose detector.

Light mesons can be studied at B factories in the following processes:

1. two-photon production:

$$
e^{+} e^{-} \rightarrow e^{+} e^{-}+\gamma^{*} \gamma^{*} \rightarrow e^{+} e^{-}+\text {hadrons }
$$

2. hadron production via initial state radiation (ISR): $e^{+} e^{-} \rightarrow e^{+} e^{-}+\gamma(I S R) \rightarrow \gamma+$ hadrons

3. hadronic (semileptonic) $\tau$-lepton decays;

4. decays of heavy $(b, c)$ mesons to light hadronic states;

5. study of light mesons in continuum.

In this report, studies of light mesons performed in the first three processes will be discussed.

\footnotetext{
ae-mail: shwartz@inp.nsk.su
}

\section{Collider and detector}

The KEKB asymmetric-energy collider operated with $8 \mathrm{GeV}$ electron and $3.5 \mathrm{GeV}$ positron beams $(\beta \gamma=0.42$ for the center-of-mass system)

The Belle detector is a large-solid-angle forward/backward asymmetric detector that combines precise magnetic spectrometry, excellent calorimetry and sophisticated particle ID ability. The schematic view of Belle is presented in Fig. 1.

The detector contains a silicon vertex detector (SVD), a 50-layer central drift chamber (CDC), an array of 1188 aerogel threshold Cherenkov counters (ACC), a barrel-like arrangement of time-of-flight scintillation counters (TOF), and an electromagnetic calorimeter (ECL) comprised of $8736 \mathrm{CsI}(\mathrm{Tl})$ crystals located inside a superconducting solenoid coil that provides a $1.5 \mathrm{~T}$ magnetic field. An iron flux return placed outside of the coil is instrumented to detect $K_{L}^{0}$ mesons and to identify muons (KLM). The detector solenoid is oriented along the $z$ axis, pointing in the direction opposite to that of the positron beam. The $r-\varphi$ plane is transverse to this axis.

\section{Two-photon collisions}

High luminosity of the B-factory provides wide possibilities to study low mass $\mathrm{C}$-even hadron states via twophoton production. In these studies properties of the light mesons as well as energy dependence of the production cross section are determined. The Feynman diagram corresponding to these processes is shown in Fig. 2.

The total cross section of the process $e^{+} e^{-} \rightarrow e^{+} e^{-}+$ $\gamma^{*} \gamma^{*} \rightarrow e^{+} e^{-}+$hadrons is given by the formula:

$$
\sigma\left(e^{+} e^{-} \rightarrow e^{+} e^{-} X\right)=\int \sigma(\gamma \gamma \rightarrow X, W) \frac{d L_{\gamma \gamma}}{d W} d W,
$$

where $d L / d W$ is an effective differential luminosity.

In the relatively low energy range, below $2-2.5 \mathrm{GeV}$, the cross section energy dependence is determined mostly 


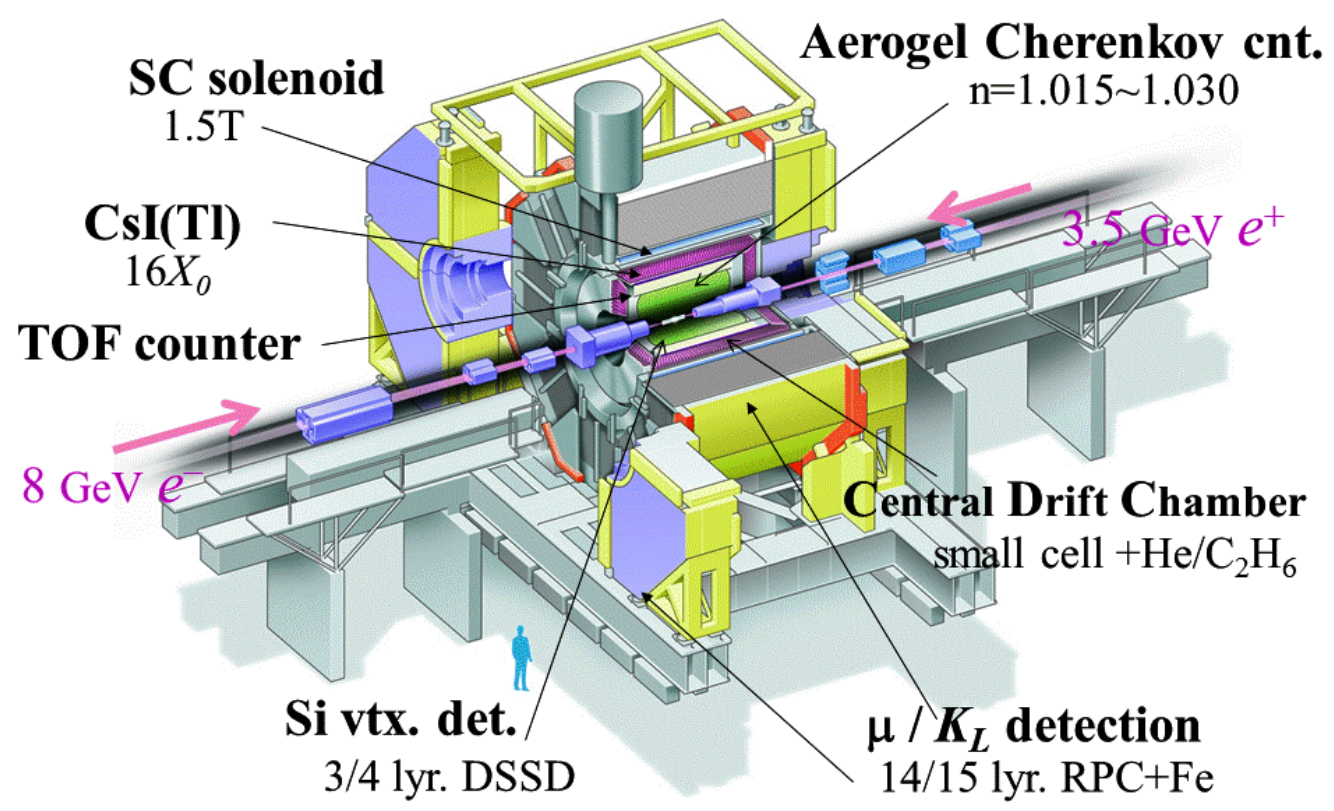

Figure 1. Schematic view of the Belle detector.

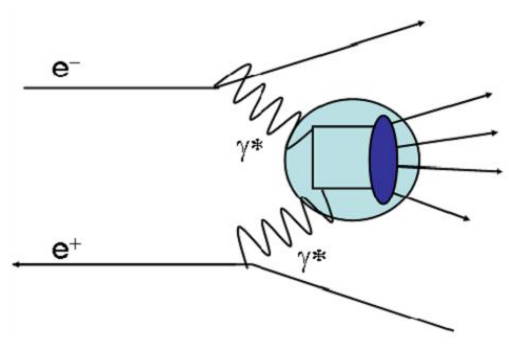

Figure 2. Two-photon production in the leading order.

by the resonances, thus the properties of various light mesons can be carefully studied. At the higher energy the cross section energy dependence is smooth and comparison of the total cross section with the QCD prediction can be performed.

Measurements of the light hadron pair production performed by the Belle collaboration are summarized in Table 1 .

A recent study of the $\gamma \gamma \rightarrow K_{S} K_{S}$ process with high statistics [9] gave precise results on the total cross section of this process presented in Fig. 3. Five resonance-like peaks are seen in the mass range below $3 \mathrm{GeV}$. These are, expectedly, $f_{2}(1270), f_{2}^{\prime}(1525), f_{J}(1710)$ and/or $a_{J}(1710)$, $f_{J}(2200)$ and/or $a_{J}(2200), f_{J}(2500)$ and/or $a_{J}(2500)$. Due to high statistics and good momentum and angular resolution of the Belle, partial wave analysis could be performed that provided a separation of the contributions of resonances with the different spin-parity quantum numbers. Evaluated parameters of resonances are listed in Table 2.

Another important study is a determination of the $\pi^{0}$ transition form factor (TFF) from the process $\gamma \gamma^{*} \rightarrow \pi^{0}$, where $\gamma^{*}$ is a virtual photon with large 4-momentum
Table 2. Parameters of resonances obtained by partial wave analysis of the $K_{S} K_{S}$ final state. The first error is statistical while the second one is systematic.

\begin{tabular}{l|c|c|c}
\hline Resonance & Mass $\left(\mathrm{MeV} / \mathrm{c}^{2}\right)$ & Width $(\mathrm{MeV})$ & $\Gamma_{\gamma \gamma} \times B(K \bar{K})(\mathrm{eV})$ \\
\hline$f_{2}^{\prime}(1525)$ & $1525.3_{-1.4-2.1}^{+1.2+3.7}$ & $82.9_{-2.2-2.0}^{+2.1+3.3}$ & $48_{-8-12}^{+67+108}$ \\
\hline$f_{0}(1710)$ & $1750_{-7-18}^{+6+29}$ & $139_{-12-50}^{+11+96}$ & $12_{-2-8}^{+3+227}$ \\
\hline$f_{2}(2200)$ & $2243_{-6-29}^{+7+3}$ & $145 \pm 12_{-34}^{+27}$ & $3.2_{-0.4-2.2}^{+0.5+1.3}$ \\
\hline$f_{0}(2500)$ & $2539 \pm 14_{-14}^{+38}$ & $274_{-61-163}^{+77+126}$ & $40_{-7-40}^{+9+17}$ \\
\hline
\end{tabular}

square, $Q^{2}$. This process is studied using events of twophoton $\pi^{0}$ production $\left(e^{+} e^{-} \rightarrow e^{+} e^{-} \pi^{0}\right)$, when one of the electrons is scattered at a large angle. The Feynman diagram of this process is shown in Fig. 4 The form factor

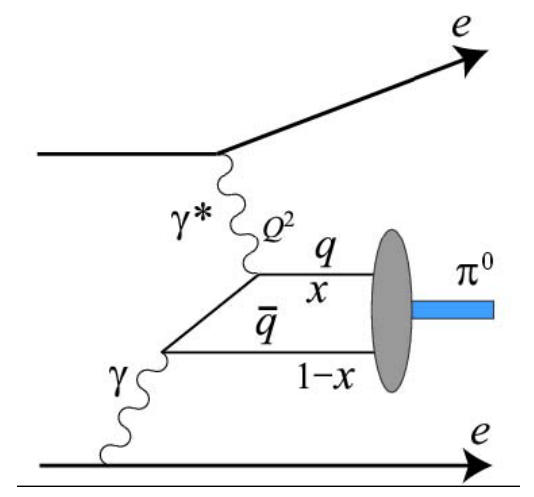

Figure 4. Single-tag $\pi^{0}$ production in two-photon process with a large- $Q^{2}$ and a small- $Q^{2}$ photon

values are calculated from the measured differential cross 
Table 1. Measurements of the light hadron pair production in the Belle experiment.

\begin{tabular}{l|c|c|c|c}
\hline Final state & Invariant mass range, $\mathrm{GeV}$ & Angular range, $\left|\cos \vartheta^{*}\right|$ & Integrated luminosity, $\mathrm{fb}^{-1}$ & reference \\
\hline$\pi^{+} \pi^{-}$ & $2.4-4.1$ & 0.6 & 88 & {$[4]$} \\
& $0.8-1.5$ & 0.6 & 86 & {$[5,6]$} \\
\hline$K^{+} K^{-}$ & $1.4-2.4$ & 0.6 & 67 & {$[7]$} \\
& $2.4-4.1$ & 0.6 & 88 & {$[4]$} \\
\hline$K_{s} K_{s}$ & $2.4-4.0$ & 0.6 & 398 & {$[8]$} \\
& $1.05-4.0$ & 0.8 & 972 & {$[9]$} \\
\hline$\pi^{0} \pi^{0}$ & $0.6-4.0$ & 0.8 & 95 & {$[10]$} \\
\hline$\eta \pi^{0}$ & $0.84-4.0$ & 0.8 & 223 & {$[11]$} \\
\hline$\eta \eta$ & $1.096-3.8$ & 1.0 & 393 & {$[12]$} \\
\hline
\end{tabular}
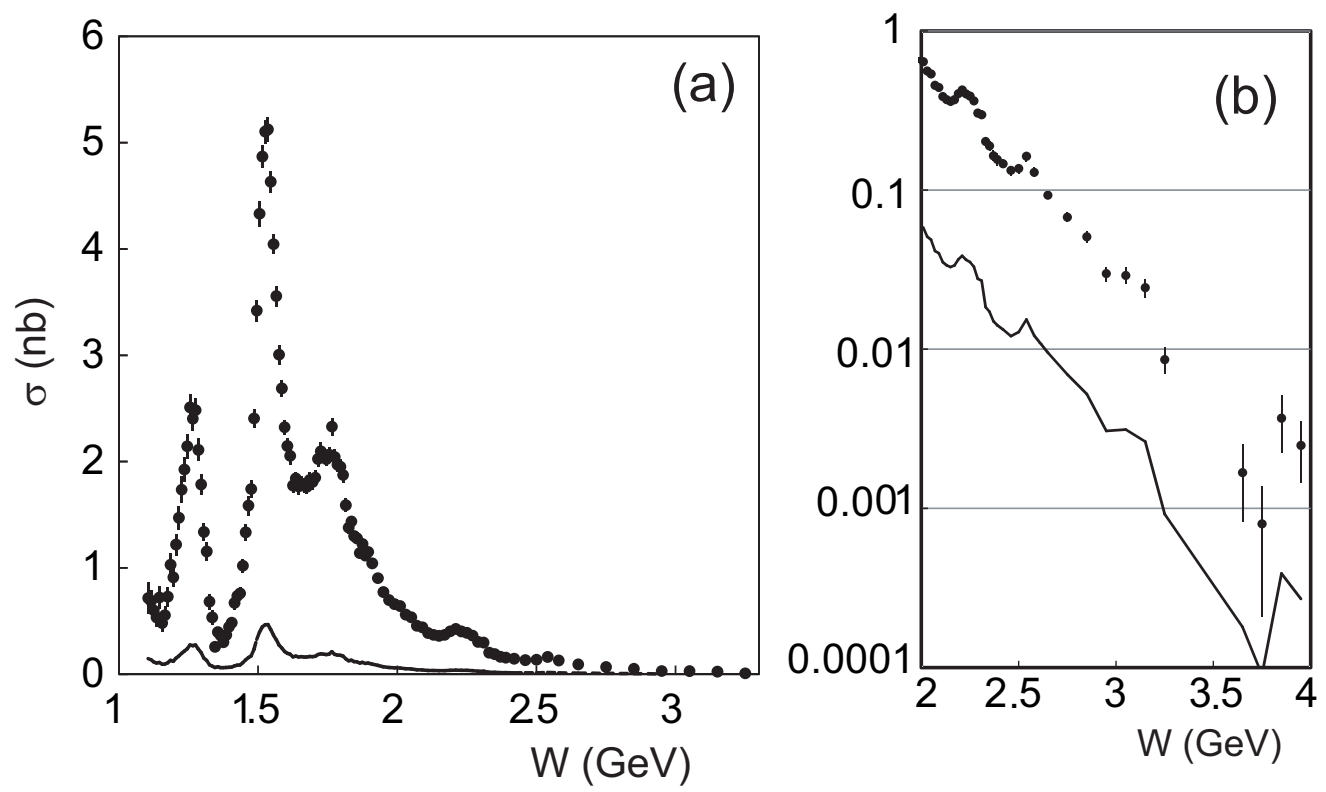

Figure 3. Cross section of $\gamma \gamma \rightarrow K_{S} K_{S}$

process measured by Belle [9] at $\left|\cos \vartheta^{*}<0.8\right| . \vartheta^{*}$ is the $K_{S}$ scattering angle in the $K_{S} K_{S}$ center-of-mass frame. The solid curve indicates systematic uncertainties.

section $d \sigma / d Q^{2}[13]$. Results of this study are presented in Fig. 5 in comparison with results from other experiments: BaBar [14], CELLO [15] and CLEO [16]. Form factor values obtained by Belle are in good agreement with QCD prediction and somewhat $(2.3 \sigma)$ lower than the BaBar results at $Q^{2}>10 \mathrm{GeV}^{2}$. In the low $Q^{2}$ range all experimental results are in good agreement.

\section{Hadronic tau decays}

Hadronic decays of $\tau$-lepton provide very clean conditions for a study of the low mass hadronic states and measurements of the light meson characteristics. The $\tau$ decay amplitude in such processes can be factorized into a purely leptonic part including $\tau$ and $v_{\tau}$ and a hadronic spectral function. In this report we describe several relevant studies.
A study of the $\tau^{-} \rightarrow \pi^{-} \pi^{0} v_{\tau}$ was performed in the Belle experiment with a data sample of only $72.2 \mathrm{fb}^{-1}$ that, nevertheless, corresponds to world highest statistics $5.6 \times 10^{6}$ decays of this mode [17]. In this study the branching fraction was measured with the world best precision, $B\left(\tau^{-} \rightarrow \pi^{-} \pi^{0} v_{\tau}\right)=(25.17 \pm 0.04 \pm 0.40) \%$. Taking into account the Conservation of Vector Current (CVC) condition, the $\pi^{-} \pi^{0}$ invariant mass spectrum was used to evaluate the pion electromagnetuc form factor, $\left|F_{\pi}\right|^{2}$. Results of this measurement are presented in Fig. 6 together with data of the CLEO [18] and ALEPH [19] experiments. A fit of the $\left|F_{\pi}\right|^{2}$ mass dependence including the $\rho, \rho^{\prime}(1450)$, and $\rho^{\prime \prime}(1700)$ resonances,

$$
F_{\pi}(s)=\frac{1}{1+\beta+\gamma}\left(B W_{\rho}+\beta \cdot B W_{\rho^{\prime}}+\gamma \cdot B W_{\rho^{\prime \prime}}\right),
$$




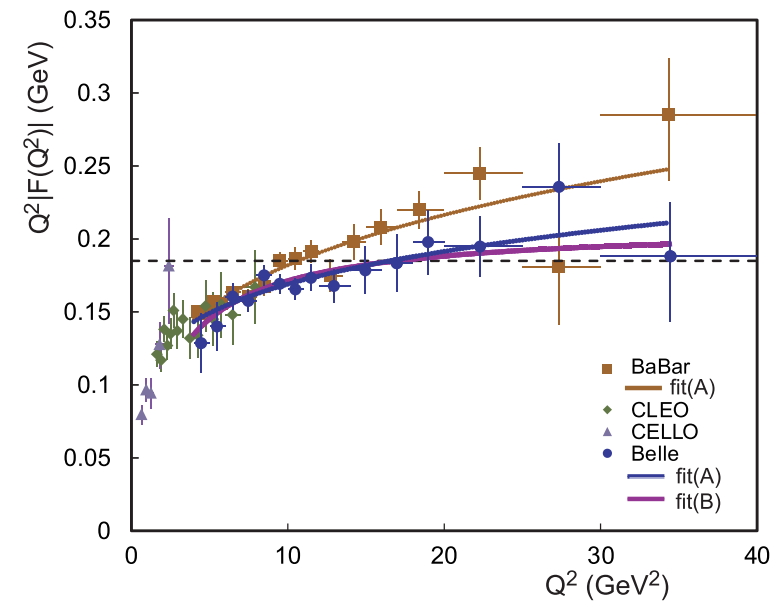

Figure 5. Values of $\pi^{0}$ transition form factor measured by Belle [13] (circles) in comparison with BaBar [14] (squares), CELLO [15] (triangles) and CLEO [16] (diamonds). Solid lines present parametrisations and the dashed line shows the asymptotic prediction from $\mathrm{pQCD}(0.185 \mathrm{GeV})$.

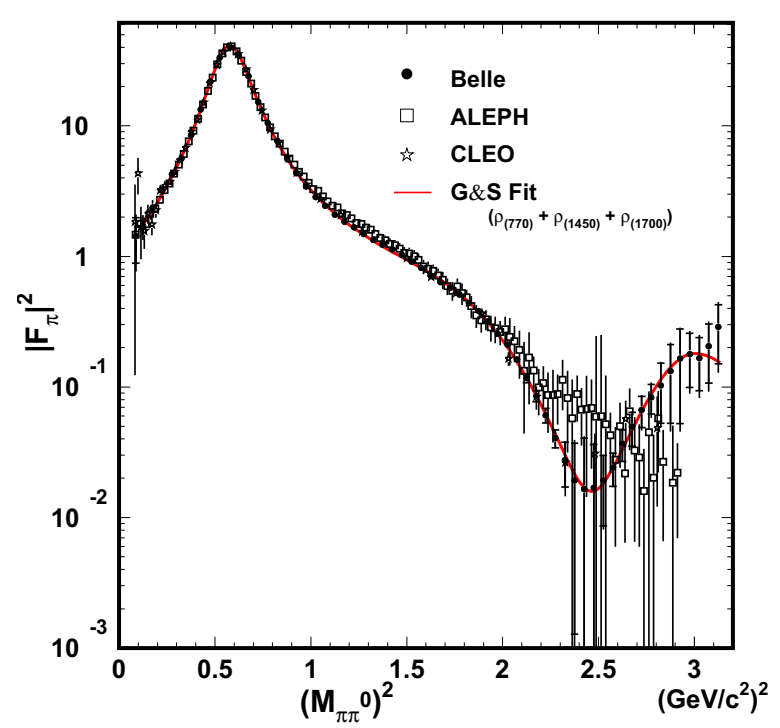

Figure 6. Pion form factor values obtained from the $\pi^{-} \pi^{0}$ invariant mass distribution in the Belle study [17] in comparison with other experiments $[18,19]$. The solid curve presents the fit to formula (2).

where $B W_{i}$ - are corresponding resonance amplitudes and $\alpha, \beta, \gamma-$ are complex constants, provided parameters of these resonances (listed in Table 3 ) with high precision.

A study of the $\tau^{-} \rightarrow K_{S} \pi^{-} v_{\tau}$ decay at Belle [20] produced not only a precise value of the branching fraction but also an accurate $K_{S} \pi^{-}$invariant mass spectrum. The main contribution to this spectral function comes from the $K^{*-}(892)$. However, to describe this spectrum the $K^{*-}(892)$ resonance is not enough and additional contributions at lower (expectedly $K_{0}^{*}(800)$ ) and higher masses $\left(K_{0}^{*}(1430)\right.$ or/and $\left.K^{*}(1410)\right)$ are needed. From the fit of the spectrum the precise values of the mass and width of
Table 3. Results of fitting the $M_{\pi \pi^{0}}^{2}$ distribution for $\tau \rightarrow \pi \pi^{0} v$ decay to the model with the $\rho(770), \rho^{\prime}(1450)$, and $\rho^{\prime \prime}(1700)$ resonances. The first error is statistical while the second one is systematic. The systematic errors include the uncertainty on the backgrounds, unfolding as well as the uncertainty of the photon energy scale. The last row gives the significance of the $\rho^{\prime \prime}(1700)$ signal.

\begin{tabular}{l|c}
\hline \hline Parameter & $\begin{array}{c}\text { Fit result } \\
\text { (all free) }\end{array}$ \\
\hline \hline$M_{\rho}, \mathrm{MeV} / c^{2}$ & $774.9 \pm 0.3 \pm 0.5$ \\
$\Gamma_{\rho}, \mathrm{MeV}$ & $148.6 \pm 0.5 \pm 1.7$ \\
$M_{\rho^{\prime}}, \mathrm{MeV} / c^{2}$ & $1428 \pm 15 \pm 26$ \\
$\Gamma_{\rho^{\prime}}, \mathrm{MeV}$ & $413 \pm 12 \pm 57$ \\
$|\beta|$ & $0.13 \pm 0.01_{-0.04}^{+0.16}$ \\
$\phi_{\beta}$, degree & $197 \pm 9_{-5}^{+50}$ \\
$M_{\rho^{\prime \prime}}, \mathrm{MeV} / c^{2}$ & $1694 \pm 41 \pm 89$ \\
$\Gamma_{\rho^{\prime \prime}}, \mathrm{MeV}$ & $135 \pm 36_{-26}^{+50}$ \\
$|\gamma|$ & $0.028 \pm 0.020_{-0.009}^{+0.059}$ \\
$\phi_{\gamma}$, degree & $-3 \pm 13_{-29}^{+136}$ \\
$|F(0)|^{2}$ & $1.02 \pm 0.01 \pm 0.04$ \\
\hline$\chi^{2} / \mathrm{NDF}$ & $65 / 51$ \\
\hline$\rho^{\prime \prime}(1700)$ signif., $\sigma$ & 7.0 \\
\hline \hline
\end{tabular}

the $K^{*-}$ meson were evaluated,

$M\left(K^{*-}(892)\right)=$

$(895.47 \pm 0.20$ (stat.) \pm 0.44 (syst. $) \pm 0.59(\bmod ).) \mathrm{MeV} / \mathrm{c}^{2}$; $\Gamma\left(K^{*-}(892)\right)=$

$(46.2 \pm 0.6$ (stat.) \pm 1.0 (syst.) \pm 0.7 (mod.) $) \mathrm{MeV}$.

The first error is statistical, the second one is systematic and the third - is an uncertainty related to the fit model. These values differ considerably from the parameters obtained in hadroproduction and close to the corresponding values for the neutral $K^{*}(892)$ mesons [21].

Recently the results of the measurements of branching fractions of $\tau$-lepton decays with one or more $K_{S}^{0}$ were published by the Belle collaboration [22]. In addition to the precise determination of seven branching fractions, the final hadronic state in the $\tau^{-} \rightarrow \pi^{-} K_{S} K_{S} \pi^{0} v_{\tau}$ decay was analyzed. It was found that main contributions to the final hadronic state in this decay are $f_{1}(1285) \pi^{-}, f_{1}(1420) \pi^{-}$ and $K^{*-} K_{S} \pi^{0}$. This is confirmed by the invariant mass spectra presented in Fig. 7.

\section{Low energy hadronic physics with ISR}

In the last years a lot of new data were obtained at the $B$ - and $\phi$-factories, by the BaBar, Belle and KLOE detectors, using initial state radiation (ISR) processes [23]. The idea of this approach is illustrated by the diagram shown in Fig. 8.

After emission of a hard photon an $e^{+} e^{-}$pair can acquire any center-of-mass energy below the energy of the 

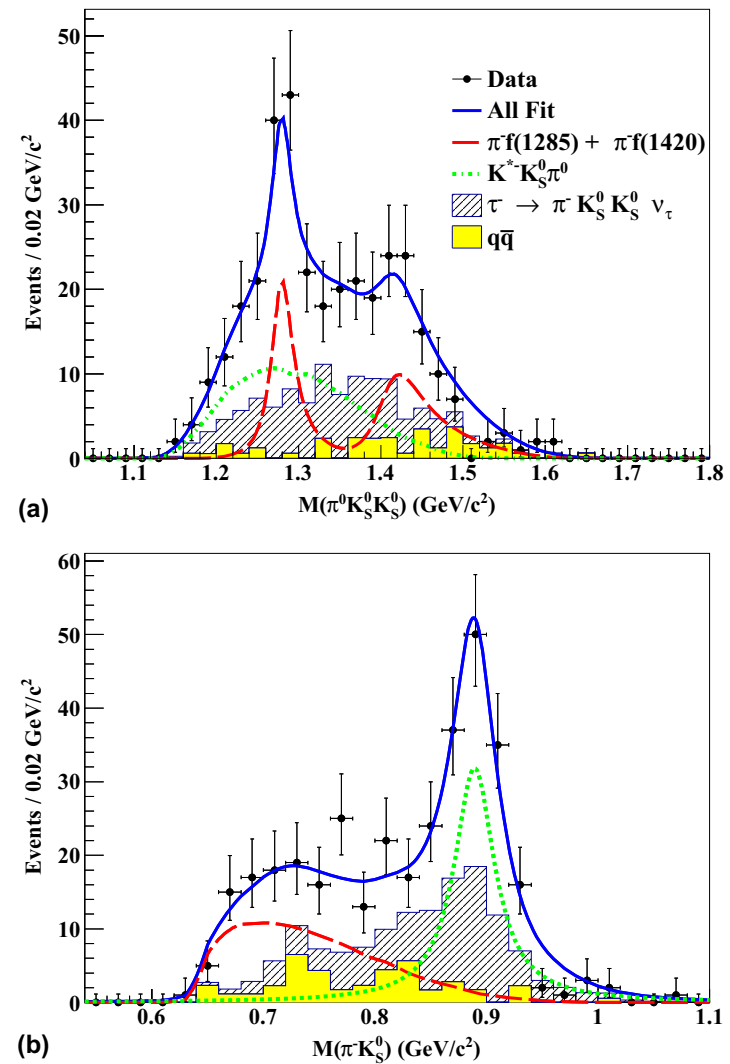

Figure 7. Hadronic invariant mass spectra in $\tau^{-} \rightarrow \pi^{-} K_{S} K_{S} \pi^{0} v_{\tau}$ decay, (a) $-\pi^{0} K_{S} K_{S}$ invariant mass, (b) $-\pi^{-} K_{S}$ invariant mass. Points with error bars are experimental data, hatched histograms show background contributions, the solid line corresponds to the best fit, dashed and dotted lines present the contributions of different intermefiate states.

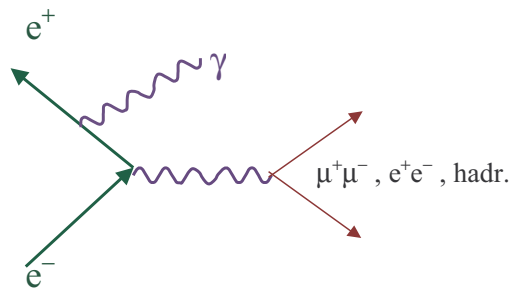

Figure 8. Diagram of the processes with initial state radiation.

experiment. That means that one can study the processes of $e^{+} e^{-}$annihilation in the entire range from the threshold to the experiment energy.

Studies of Belle in this field are mostly related to the charm sector. However, interesting results were also obtained for the processes $e^{+} e^{-} \rightarrow \phi \pi^{+} \pi^{-}$and $e^{+} e^{-} \rightarrow$ $\phi f_{0}(980)$ in the energy range from 1.5 to $3 \mathrm{GeV}$ with the total integrated luminosity of $673 \mathrm{fb}^{-1}$ [24]. Measured cross sections are shown in Fig. 9. Systematic uncertainties are $8.6 \%$ and $6.9 \%$ for the $\phi \pi^{+} \pi^{-}$and $\phi f_{0}(980)$ cross sections, respectively. Parameters of the $\phi(1680)$ and $Y(2175)$ were measured to be:

$M(\phi(1680))=(1689 \pm 7 \pm 10) \mathrm{MeV} / \mathrm{c}^{2}$,

$\Gamma(\phi(1680))=(211 \pm 14 \pm 19) \mathrm{MeV}$,
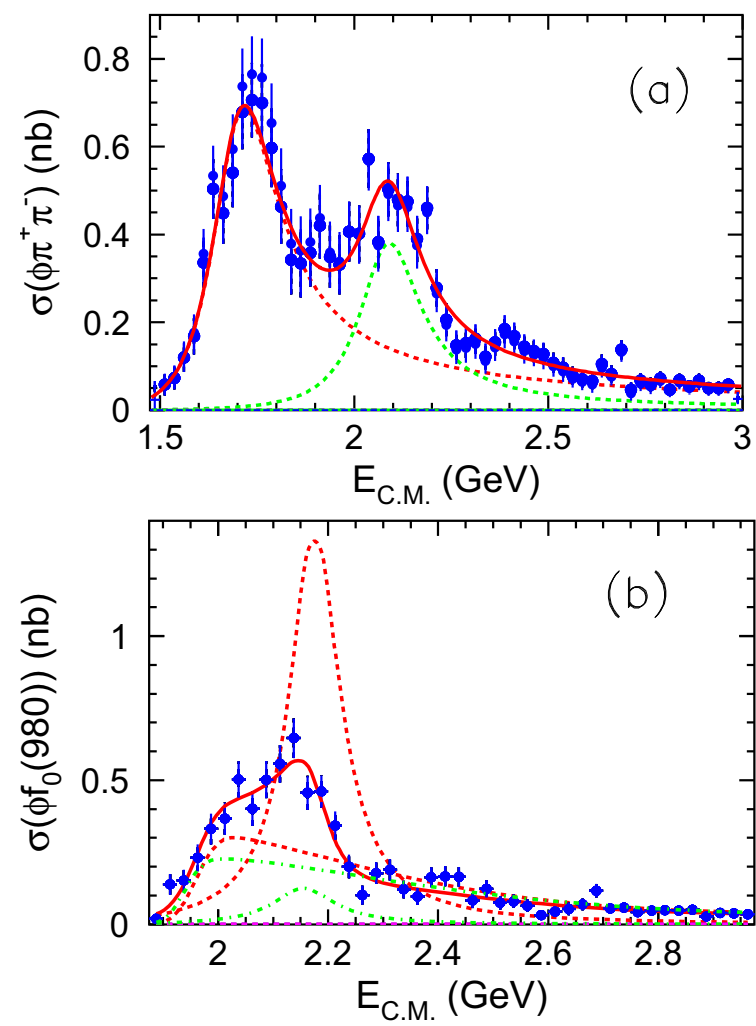

Figure 9. Fit to: (a) $e^{+} e^{-} \rightarrow \phi \pi^{+} \pi^{-}$cross section with two incoherent Breit-Wigner (BW) functions, one for the $\phi(1680)$ and the other for the $Y(2175)$ and (b) $e^{+} e^{-} \rightarrow \phi f_{0}(980)$ cross section with a single BW function that interferes with a non-resonant component. The curves show the projections from the best fit and the contribution from each component. In (b), the dashed curves are for the destructive interference solution and the dot-dashed curves for the constructive interference solution

$$
\begin{aligned}
& M(Y(2175))=\left(2079 \pm 13_{-28}^{+79}\right) \mathrm{MeV} / \mathrm{c}^{2} \\
& \Gamma(Y(2175))=\left(192 \pm 23_{-61}^{+25}\right) \mathrm{MeV}
\end{aligned}
$$

\section{Super KEKB and Belle II}

At present a new advanced collider, SuperKEKB, with a project luminosity of $8 \times 10^{35} \mathrm{~cm}^{-2} \mathrm{~s}^{-1}$ is under construction at KEK.

The new SuperKEKB collider is placed in the KEKB tunnel utilizing many elements of the previous B-factory. The KEKB cells are kept in the High Energy Ring (HER). Dipole magnets in the Low Energy Ring (LER) will be replaced, while other main magnets in the LER arcs are reused. The LER beam pipe will be completely replaced by a new one with ante-chambers. The injector for KEKB is upgraded as well. A special damping ring is constructed to produce high intensive positron beams with low emittance.

The basic idea of the new collider is the so called "Nano-Beam" scheme, first proposed in [25]. Main parameters of the new collider are listed in Table 4 in comparison with those achieved by KEKB. At present most of the collider elements are produced and installed in the tunnel. 
Table 4. Comparison of the main parameters of SuperKEKB and previous KEKB colliders

\author{
Energy, $\mathrm{GeV}\left(e^{+} / e^{-}\right)$ \\ Beam current, A \\ Vertical $\beta$ function at IP, mm \\ Beam-beam parameter, $\xi_{y}$ \\ Beam sizes $\left(\sigma_{x}, / \sigma_{y}\right), \mu \mathrm{m}$ \\ Luminosity, $10^{34} \mathrm{~cm}^{-2} \mathrm{~s}^{-1}$
}

$\begin{array}{cc}\text { KEKB (achieved) } & \text { SuperKEKB } \\ 3.5 / 8.0 & 4.0 / 7.0 \\ 1.64 / 1.19 & 3.6 / 2.6 \\ 5.9 / 5.9 & 0.27 / 0.30 \\ 0.129 / 0.090 & 0.09 / 0.081 \\ 100 / 2 & 10 / 0.06 \\ 2.11 & 80\end{array}$

Belle II detector will produce new important information in various fields of particle physics. This information will be complementary to that obtained in the experiments at LHC. At present, the superKEKB/Belle II project is under construction and we can hope for new exciting results in the next decade.

\section{Acknowledgments}

I would like to thank organisers for the kind invitation to this impressive Conference as well as for their help and hospitality in Messina.

This work is supported in part by the Russian Education and Science Ministry, by agreement 14.B37.21.07777 and by the Russian Fund for Basic Research grant 12-0201296-a.

\section{References}

[1] A. Abashian et al., Nucl. Instr. and Meth., A479, 117 (2002).

[2] S. Kurokawa et al., Nucl. Instr. and Meth., A499, 1 (2003).

[3] Belle Collab. (A. Abashian et al.), Phys. Rev. Lett. 86, 2509 (2001);

BABAR Collaboration (B. Aubert et al. ), Phys. Rev. Lett. 86, 2515 (2001).

[4] Belle Collaboration (H. Nakazawa et al. ), Phys. Lett. B615, 39 (2005).

[5] T. Mori et al., Phys. Rev. Phys. Rev. D75, 051101 (2007).

[6] T. Mori et al., J. Phys. Soc. Jpn 76, 074102 (2007).

[7] Belle Collaboration (K. Abe et al. ), Eur. Phys. J. C32, 323 (2004).

[8] Belle Collaboration (W.T. Chen et al. ), Phys. Lett. B651, 15 (2007).

[9] Belle Collaboration (S. Uehara et al. ), Prog. Theor. Exp. Phys. 123C01 (2013).

[10] S. Uehara et al., Phys. Rev. D78, 052004 (2008).

[11] S. Uehara et al., Phys. Rev. D80, 032001 (2009).

[12] S. Uehara et al., Phys. Rev. D82, 114031 (2010).

[13] S. Uehara et al., Phys. Rev. D86, 092007 (2012).

[14] B. Aubert et al., Phys. Rev. D80, 052002 (2009).

[15] H.J. Behrend et al. (CELLO Collaboration), Z. Phys. C 49, 401 (1991).

[16] J. Gronberg et al. (CLEO Collaboration), Phys. Rev. D57, 33 (1998). he broad field of research will be opened by thr superB-factory. It is clear that the superB-factory with the 
[17] M. Fujikawa et al. (Belle Collaboration), Phys. Rev. D 78, 072006 (2008).

[18] S. Anderson et al. (CLEO Collaboration), Phys. Rev. D 61, 112002 (2000).

[19] S. Schael et al. (ALEPH Collaboration), Phys. Rep. 421, 191 (2005).

[20] Belle Collaboration (D. Epifanov et al.), Phys. Lett. B654, 65 (2007).

[21] Particle Data Group (K.A. Olive et al.), Chin. Phys. C 38 (2014).

[22] S. Ryu et al. (Belle Collaboration), Phys. Rev. D 89, 072009 (2014).
[23] V.N. Baier, V.A.Khoze, Sov. Phys. JETP 21, 1145 (1965); S. Binner, H.J. Kuhn, K. Melnikov, Phys. Lett.B 459, 279 (1999); M.Benayoun et al., Mod. Phys. Lett. A 14, 2605 (1999).

[24] Z. Q. Liu et al. (Belle Collaboration), Phys. Rev. Lett. 110, 252002 (2013).

[25] P. Raimondi, Talk, given at the $2 d \quad$ SuperB workshop, Frascati, http://www.lnf.infn.it/conference/superb06/talks/raimondi1.ppt, 2006.

[26] T. Abe et al., Belle II technical design report, KEK Report 2010-1 (2010). 